\title{
Atrial Fibrillation and Use of Rivaroxaban: Performance of the Prothrombin Time / INR as a Function of Time After Blood Collection
}

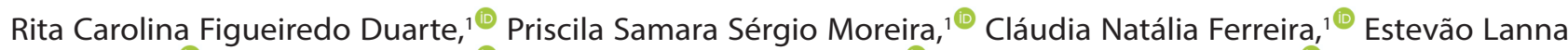
Figueiredo, ${ }^{\circledR}$ Eduardo Sternick, ${ }^{3 \oplus}$ Francisco Rezende Silveira, ${ }^{4}{ }^{\circledR}$ Luan Carlos Vieira Alves, ${ }^{1}$ Ana Paula Lucas Mota, ${ }^{\circledR}$ Edna Afonso Reis, ${ }^{5}{ }^{\circledR}$ Maria das Graças Carvalho, ${ }^{1 \oplus}$ Helton José dos Reis ${ }^{1 \odot}$

Universidade Federal de Minas Gerais,' Belo Horizonte, MG - Brazil.

Lifecenter Hospital, ${ }^{2}$ Belo Horizonte, MG - Brazil.

Faculdade de Ciências Médicas de Minas Gerais, ${ }^{3}$ Belo Horizonte, MG - Brazil.

Semper Hospital, ${ }^{4}$ Belo Horizonte, MG - Brazil.

Department of Statistics- ICEx/UFMG, ${ }^{5}$ Belo Horizonte, MG - Brazil.

\section{Abstract}

Background: Traditionally, the most effective therapy in the prevention of stroke in patients with atrial fibrillation (AF) has been oral anticoagulation with vitamin $\mathrm{K}$ inhibitors, particularly warfarin, whose disadvantages and adverse effects have led to their replacement by "direct oral anticoagulants", as factor X inhibitor.

Objectives: This study aimed to conduct a brief approach on atrial fibrillation (AF) and use of Rivaroxaban, and to comparatively evaluate the prothrombin time / International Normalized Ratio (PT/INR) in patients with AF in use of this oral anticoagulant, depending on the time elapsed between the last administration of the drug and the time of blood sample venipuncture.

Methods: We evaluated 34 patients with AF in use of Rivaroxaban by using PT / INR, distributed into a subgroup with blood collection time $\leq 12$ hours $(n=7)$ and $>12$ hours after the last drug intake $(n=27)$. Mann-Whitney test was used to compare the groups and $\mathrm{p}<0.05$ was considered significant.

Results: An analysis as a function of time between the Rivaroxaban intake and blood collection, revealed that PT / INR suffers the greatest effect up to 12 hours after ingestion of the drug, dropping to levels close to normal in subsequent hours before the next dose.

Conclusion: We concluded that, in contrast to warfarin, the knowledge of the time interval between drug intake and blood collection from patients taking Rivaroxaban is essential to properly interpret a laboratory test to assess hemostasis, particularly PT and its derivatives. (Int J Cardiovasc Sci. 2021; 34(2):116-121)

Keywords: Atrial Fibrillation; Stroke; Embolism; Rivaroxaban; Warfarin; Anticoagulants; Hemorrhage/ physiopathology; Risk Assessment; Blood Coagulation.

\section{Introduction}

Patients with atrial fibrillation (AF) present hemostatic system changes that contribute to an approximately 5-fold increased risk of stroke. According to the American Heart Association, ${ }^{1}$ the American College of Chest Physicians, ${ }^{2}$ and the European Society of Cardiology (ESC), ${ }^{3}$ recommendations for antithrombotic therapy of patients with non-valvular and chronic AF should be based fundamentally on the measurement of the prothrombotic risk factors of each patient, which determines the existence of different levels of risk among the different groups of patients. Traditionally, the most effective therapy in preventing stroke in patients with AF has been oral anticoagulation with vitamin $\mathrm{K}$ inhibitors - also referred to as vitamin $\mathrm{K}$ antagonists (VKAs), especially warfarin. Although this drug has an established efficacy, a number of genetic and environmental factors - such as

\section{Mailing Address: Maria das Graças Carvalho}


diet and concomitant drug use - influence the patient's response to the drug, leading to a high risk of bleeding and, thus, requiring frequent monitoring of coagulation. ${ }^{4}$ For this purpose, the International Normalized Ratio (INR), obtained using the International Sensitivity Index (ISI), has been used based on the comparison of the sensitivity of commercial thromboplastin in relation to WHO standard thromboplastin.

A number of drawbacks related to the need for strict laboratory monitoring and the occurrence of hemorrhages in the course of warfarin anticoagulation have limited its use in clinical practice and encouraged its replacement with newly developed oral anticoagulants including direct thrombin inhibitors and factor Xa inhibitors, ${ }^{4}$ called direct oral anticoagulants (DOACs). ${ }^{5}$

Four DOACs (dabigatran, rivaroxaban, apixaban and edoxaban) have become available for treatment of patients with AF. These drugs have a comparable efficacy to warfarin in large randomized controlled trials and were found to pose a lower risk of intracranial bleeding. ${ }^{6}$ DOACs have been widely used for primary or secondary prevention of stroke in patients with $\mathrm{AF}$, while $\mathrm{AF}$ ablation under uninterrupted warfarin or rivaroxaban has shown a safety profile. ${ }^{7,8,9}$ However, there is no evidence of benefit of DOACs in patients with mechanical prosthetic valves or moderate/severe mitral stenosis. ${ }^{6}$

Direct thrombin inhibitors and factor Xa inhibitors do not usually require laboratory monitoring. However, standardized and well-established tests for laboratory evaluation of the anticoagulant effect of these drugs are not yet available when needed in special occasions in the usual medical practice. ${ }^{10}$ Therefore, greater knowledge of this topic should be sought by evaluating hemostatic tests that are routinely used in clinical laboratories, such as prothrombin time (PT), in patients using direct oral anticoagulants since such hemostatic parameters may be extensive and variably affected by these drugs. ${ }^{11}$

Specific tests include the anti-factor Xa chromogenic assay, which has been suggested for laboratory evaluation of Rivaroxaban and is able to estimate the plasma concentration of the drug when specific calibrators are used. This method has been shown to be adequate for determination of Rivaroxaban over a wide concentration range, but it is not routinely available in most laboratories. ${ }^{12,13}$

In order to provide a small contribution in this context, the present study aimed to comparatively evaluate PT/ INR in patients with AF using Rivaroxaban, according to the time elapsed between the last administration of the drug and the time of collection of blood samples from the patient.

\section{Methods}

We evaluated 34 patients with AF using Rivaroxaban

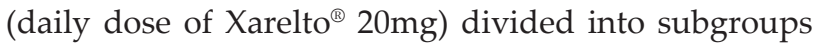
with collection time $\leq 12$ hours $(n=7)$ and collection time $>12$ hours after the last administration of the drug $(n=27)$. The use of the drug was investigated and confirmed by patient self-report. The project was approved by the Research Ethics Committee of the Universidade Federal de Minas Gerais and the Lifecenter Hospital Ethics Committee. Individuals selected as participants were informed of the research objectives and signed a free and informed consent form prior to blood collection. For each participant, a standardized clinical record was also filled out, whose data were essential for the analysis of results of the present study. The sample size of both the study group and its subgroups was defined for convenience.

Blood samples of control individuals (not in use of anticoagulant or other medication with effects on the hemostatic system), who were selected by the medical team or by the researchers, were used in parallel to test the quality of the reagents for PT, and whose results were found within normal limits according to the manufacturer.

Samples of $10 \mathrm{~mL}$ of venous blood $(5 \mathrm{~mL}$ in $3.2 \%$ sodium citrate and $5 \mathrm{~mL}$ without additive) were collected using the Vacutainer ${ }^{\circledR}$ System (Becton-Dickinson) tubes in the morning, requiring a 12-hour fast. Blood samples were rapidly centrifuged at $3500 \mathrm{rpm}$ for 15 minutes in room temperature. Plasma samples were transferred to plastic tubes and then centrifuged again under the same conditions to ensure platelet-poor plasma $\left(<10000 / \mathrm{mm}^{3}\right)$. The serum and plasma obtained were divided into several aliquots and stored at $-80^{\circ} \mathrm{C}$ until use.

Regarding the inclusion criteria, the case group of the present study included patients with paroxysmal, persistent, or permanent non-valvular AF at medium or high risk of thromboembolic events (CHADS2 $\geq 2$ ), men and women, aged over 18 years and with a history of AF of any duration, documented by electrocardiogram and/or 2-dimensional doppler echocardiogram within 12 months prior, and for whom anticoagulation was indicated. Patients with transient AF secondary to other reversible disorders (thyrotoxicosis, cardiac or 
thoracic surgery, pneumonia, and severe anemia), situations in which chronic anticoagulation therapy was interrupted during the duration of the present study; any contraindication for anticoagulant agents; conditions associated with elevated risk of bleeding (intracranial, intraocular, spinal, retroperitoneal, and intra-articular bleeding, gastrointestinal bleeding, or active ulcer within the previous year; recent severe trauma, major surgery and active infective endocarditis), uncontrolled hypertension (blood pressure greater than $170 / 100 \mathrm{mmHg}$ ), or acquired or hereditary hemorrhagic disorders; association of antiplatelet therapy or fibrinolytic therapy with the use of oral anticoagulants; amiodarone therapy, verapamil, quinidine, ketoconazole, ritonavir, corticosteroids, anti-inflammatories, heparin, fondaparinux, or hormone replacement therapy; hepatic, malignant, autoimmune, thyroid, and infectious diseases; severe renal insufficiency (creatinine clearance less than $30 \mathrm{~mL} / \mathrm{min}$ ); alcoholism; pregnancy, or any relevant laboratory or clinical change detected by the physician investigation were excluded.

\section{PT and INR}

PT was measured by automated coagulometric method, using the Destiny Max ${ }^{\mathrm{TM}}$ diagnostic system, and TriniCLOT PT Excel S, ISI = 1.2 (TCoag, Wicklow, Ireland) as a reagent.

\section{Biochemical Characterization of Participants}

In order to verify the presence and absence of comorbidities that could influence the results of PT/ INR, a biochemical characterization of participants was undertaken, including determination of alanine aminotransferase (ALT), aspartate aminotransferase (AST), gamma glutamyltransferase (GGT), creatinine, triglycerides, total cholesterol, high-density lipoprotein (HDL), low-density lipoprotein (LDL), and C-reactive protein (CRP).

\section{Statistical Analysis}

Statistical analyses were performed by the MiniTab program (version 17). Graphs were plotted (scatter plotting) using GraphPad Prism ${ }^{\circledR}$, version 6.07. Because PT and INR did not show a normal distribution, the results were presented as median and interquartile range. The Mann-Whitney test was used to compare the groups, and $p<0.05$ was considered significant.

\section{Results}

Regarding the biochemical characterization of patients performed to rule out comorbidities that could influence the results of PT and its derived parameters, no significant changes were detected, that is, hepatic, renal and lipid profiles were within the reference limits.

The comparison of the parameters of PT and INR in patients with AF using Rivaroxaban as a function of the time elapsed between the last dose of the drug and the moment of blood collection is presented in Table 1 .

$\mathrm{PT}$, in seconds, was considerably higher in patients whose Rivaroxaban administration was $\leq 12 \mathrm{~h}$, suggesting that the test was sensitive to detect anticoagulant effect. As a consequence, prothrombin activity, evaluated in terms of median and interquartile range was lower in this group. INR also appeared to be different between subgroups and was significantly higher in the $\leq 12 \mathrm{~h}$ subgroup, although this ratio is not considered a reliable mode of expression in the context of Rivaroxaban use. The results are shown in Figure 1 and Figure 2.

\section{Discussion}

Markers of hepatic and renal function and lipid profile were evaluated in the present study, whose results were normal. This fact is important since it reflects that the results of PT/INR were not changed by liver or kidney diseases. Thus, changes detected in PT/INR are very likely to be attributed to the use of Rivaroxaban, since the patients also did not present other characteristics that could compromise the results, such as weight extremes, for example.

Table 1 - Prothrombin time in patients with atrial fibrillation using rivaroxaban, according to the time between administration of the drug and blood collection

\begin{tabular}{|c|c|c|c|}
\hline & $\begin{array}{c}\text { Time } \leq 12 h \\
(n=7)\end{array}$ & $\begin{array}{c}\text { Time }>12 h \\
(n=27)\end{array}$ & $\begin{array}{c}p \\
\text { value }\end{array}$ \\
\hline PT (s) & $32.9(15.5)$ & $20.5(7.4)$ & 0.003 \\
\hline INR & $2.69(0.45)$ & $1.54(0.56)$ & 0.003 \\
\hline \multicolumn{4}{|c|}{$\begin{array}{l}{ }^{*} p<0.05 . \text { Mann Whitney Test. Values presented as median and } \\
\text { interquartile range. PT(s): prothrombin time (seconds). INR: } \\
\text { International Normalized Ratio. }\end{array}$} \\
\hline
\end{tabular}




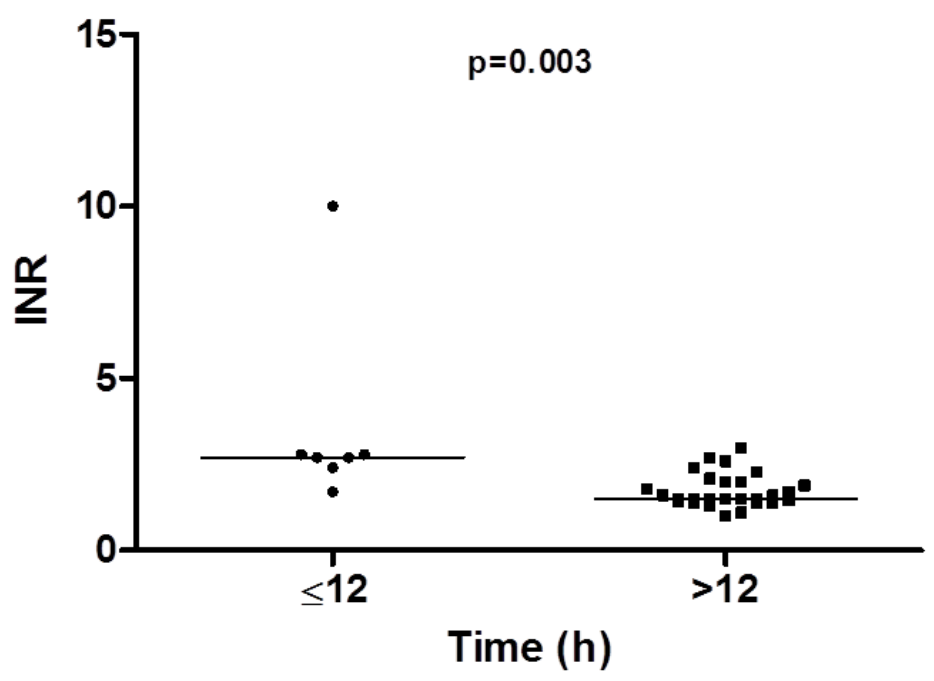

Figure 1 - Prothrombin time of patients in use of Rivaroxaban, as a function of time of blood collection after administration of the drug. The horizontal lines represent the median of each subgroup (Mann Whitney Test).

INR: International Normalized Ratio.

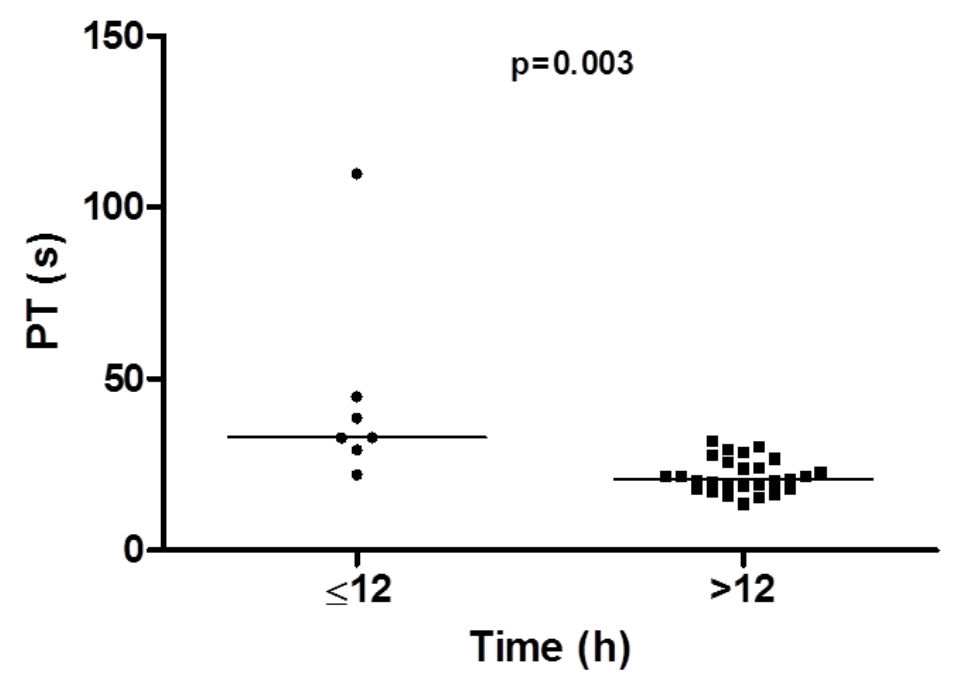

Figure 2 - INR of patients in use of Rivaroxaban, as a function of time of blood collection, after administration of the drug. The horizontal lines represent the median of each subgroup (Mann Whitney Test).

PT(s): prothrombin time (seconds). 
The levels of C-reactive protein (CRP) were also evaluated, which were not different between the subgroups, indicating no differences in the inflammatory status for the participants of this study. Regarding the use of other drugs, such as statins or antihypertensives, the limited sample size prevented appropriate analyses.

The prolongation of PT observed in the present study in patients using Rivaroxaban, in relation to those found for healthy non-medicated individuals is in line with previous studies reported in the literature, such as that by Douxfils et al. ${ }^{9}$ According to these studies, PT can be used as a screening test to assess bleeding risk, although more specific tests using calibrators should be applied to confirm the plasma concentration of Rivaroxaban. In this regard, ISTH basically defines 2 types of tests for the evaluation of direct oral anticoagulants: simple semiquantitative tests available in most laboratories, and quantitative tests capable of accurately reporting levels of the drug. The effect of Rivaroxaban has been investigated on both types of tests in order to provide evidence to support the standardization of the laboratory evaluation of this drug. It is emphasized that the use of Rivaroxaban does not require frequent monitoring or periodic dose adjustment, however, the need for standardized sensitive and safe laboratory tests (qualitative and quantitative) is unquestionable, which allows the evaluation of the degree of anticoagulation or determination of optimal dose in specific situations. ${ }^{14}$ These include, for example, evaluation of special populations of patients, such as those with hepatic or renal insufficiency, weight in extreme ranges, imminent surgery, and cases of therapeutic failure or suspected non-adherence to treatment. ${ }^{10}$ In these circumstances, it is necessary to know the performance of the tests available in the investigation of the hemostatic status of the individual.

Although a very limited number of samples were assayed, our findings indicate that at the plasma peak of the drug (around 2 to 3 hours), PT is quite sensitive to evaluate the effect of Rivaroxaban.

However, close to the administration of the subsequent dose (after 24 hours), this test is no longer significantly prolonged as a function of the reduced anticoagulant plasma levels. It should be noted that the result of PT is largely influenced by the quality of thromboplastin employed, whose variability is not corrected when expressing the result in INR. The development of a validated sensitivity index for Rivaroxaban and the consequent use of an INR specific for this anticoagulant have already been proposed to minimize the variability among the reagents. ${ }^{15}$
In summary, the results of PT and its derivatives in the present study suggest a sensitivity of the test to detect the anticoagulant effect up to 12 hours after drug ingestion, dropping to values close to normal in the subsequent hours before the next dose. However, for a greater test reliability it would be necessary to develop a thromboplastin sensitivity index to be used, validated for Rivaroxaban.

\section{Conclusion}

In agreement with Douxfils et al. ${ }^{14}$ and Samama et al. ${ }^{16}$, it is concluded that, in contrast to warfarin, knowledge of the time interval between drug administration and blood collection in patients using Rivaroxaban is essential to interpret a laboratory test that evaluates hemostasis, particularly PT and its derivatives. Therefore, it is essential to be aware that the results of PT will be affected as a function of the time of blood collection after taking the drug.

\section{Acknowledgment}

This work was supported by Conselho Nacional de Desenvolvimento Científico e Tecnológico - CNPq (Grant number 477147/2010-0), Fundação de Amparo à Pesquisa do Estado de Minas Gerais - FAPEMIG, Ministério da Saúde - MS and Secretária de Estado da Saúde de Minas Gerais SES-MG. We also thank the patients who generously participated in this study.

\section{Author Contributions}

Conception and design of the research: Duarte RCF, Moreira PSS, Ferreira CN, Carvalho MG. Acquisition of data: Duarte RCF, Moreira PSS, Figueiredo EL, Sternick E, Silveira FR, Mota APL. Analysis and interpretation of the data: Duarte RCF, Moreira PSS, Ferreira CN, Alves LCV, Carvalho MG. Statistical analysis: Reis, EA, Duarte RCF, Moreira PSS. Obtaining financing: Carvalho MG, Reis HJ. Writing of the manuscript: Duarte RCF, Moreira PSS. Critical revision of the manuscript for intellectual content: Carvalho MG, Reis HJ.

\section{Potential Conflict of Interest}

No potential conflict of interest relevant to this article was reported. 


\section{Sources of Funding}

This study was funded by Conselho Nacional de Desenvolvimento Científico Tecnológico CNPq- Brazil.

\section{Study Association}

This article is part of the thesis of master submitted by Priscila Samara Sérgio Moreira, from Universidade Federal de Minas Gerais - Faculdade de Farmácia.

\section{References}

1. January CT, Wann LS, Alpert JS, Calkins H, Cigarroa JE, Cleveland Jr. JC, et al. ACC/AHA Task Force Members, 2014 AHA/ACC/HRS Guideline for the Management of Patients with Atrial Fibrillation: Executive Summary: A Report of the American College of Cardiology/ American Heart Association Task Force on Practice Guidelines and the Heart Rhythm Society. J Am Coll Cardiol. 2014; 64(21):2246-80.

2. You JJ, Singer DE, Howard PA, Lane DA, Eckman MH, Fang MC, et al. American College of Chest Physicians. Antithrombotic Therapy for Atrial Fibrillation: Antithrombotic Therapy and Prevention of Thrombosis, 9th ed: American College of Chest Physicians Evidence-Based Clinical Practice Guidelines. Chest. 2012;141(2):e531S-575S

3. Camm AJ, Lip GY, De Caterina R, Savelieva I, Atar D, Hohnloser SH, et al. ESC Committee for Practice Guidelines (CPG), 2012 focused update of the ESC Guidelines for the management of atrial fibrillation: an update of the 2010 ESC Guidelines for the management of atrial fibrillation. Developed with the special contribution of the European Heart Rhythm Association. Eur Heart. J. 2012;33(21):2719-47.

4. Al-Khatib SM, Alexander JH, Lopes RD, Mahaffey KW, Patel MR, Granger CB. Promise of Factor Xa Inhibition in Atrial Fibrillation. Curr Cardiol Rep. 2012;14(1):70-8.

5. Barnes GD, Ageno W, Ansell J, Kaatz S. Subcommittee on the Control of Anticoagulation of the International Society on Thrombosis and Haemostasis, Recommendation on the nomenclature for oral anticoagulants: communication from the SSC of the ISTH. J Thromb Haemost. 2015;13(6):1154-6.

6. Ferrari F, da Silveira AD, Martins VM, Franzoni LT, Zimerman LI, Stein R. Direct-Acting Oral Anticoagulants in Atrial Fibrillation: What's New in the Literature. Cardiol Rev. 2020 Apr 23. ID mdl 32332235

7. Silva MA, Futuro GMC, Merçon ES, Vasconcelos D, Agrizzi RS, Elias Neto J, Kuniyoshi R. Safety of Catheter Ablation of Atrial Fibrillation Under Uninterrupted Rivaroxaban Use. Arq Bras Cardiol. 2020;114(3):435-42.

\section{Ethics Approval and Consent to Participate}

This study was approved by the Ethics Committee of the Universidade Federal de Minas Gerais (COEP-UFMG) under the protocol number CAAE 12603413.0.0000.5149. All the procedures in this study were in accordance with the 1975 Helsinki Declaration, updated in 2013. Informed consent was obtained from all participants included in the study.

8. Kohsaka S, Katada J, Saito K, Jenkins A, Li B, Mardekian J, Terayama Y. Safety and effectiveness of non-vitamin $\mathrm{K}$ oral anticoagulants versus warfarin in real-world patients with non-valvular atrial fibrillation: a retrospective analysis of contemporary Japanese administrative claims data. Open Heart. 2020;7(1):e001232.

9. Salmasi S, Loewen PS, Tandun R, Andrade JG, De Vera MA. Adherence to oral anticoagulants among patients with atrial fibrillation: a systematic review and meta-analysis of observational studies. BMJ Open. 2020;10 (4):e034778.

10. Favaloro EJ, Lippi G. The new oral anticoagulants and the future of haemostasis laboratory testing. Biochem Med. 2012;22(3):329-41.

11. Tripodi A. The Laboratory and the New Oral Anticoagulants. Clin Chem. 2013; 59 (2): 353-362.

12. Douxfils J, Mani H, Minet V, Devalet B, Chatelain B, Dogné JM, et al. Non-VKA Oral Anticoagulants: Accurate Measurement of Plasma Drug Concentrations. Biomed. Res Int. 2015(Special issue):345138.

13. Sarode R. Direct oral anticoagulant monitoring: what laboratory tests are available to guide us? Hematology Am Soc Hematol Educ Program. 2019; 2019 (1):194-7.

14. Douxfils J, Mullier F, Loosen C, Chatelain C, Chatelain B, Dogné JM. Assessment of the impact of rivaroxaban on coagulation assays: laboratory recommendations for the monitoring of rivaroxaban and review of the literature. Thromb Res. 2012;130(6):956-66.

15. Tripodi A, Chantarangukul V, Guinet V, Samama MM. The International Normalized Ratio calibrated for rivaroxaban has the potential to normalize prothrombin time results for rivaroxaban-treated patients: results of an in vitro study. J Thromb Haemost. 2011;9(1):226-8.

16. Samama MM, Contant G, Spiro TE, Perzborn E, Le Flem L, Guinet C, et al. Laboratory assessment of rivaroxaban: a review, Thromb. J. 2013;11(1):11. 